\title{
Design of synchronous optimal control strategy based on double motor drive precision air suspension platform
}

\author{
Ling Xiang ${ }^{1}$, Zhang Yu ${ }^{1}$, Guo Xiao-kun ${ }^{2}$ and Li Xi-feng ${ }^{1}$ \\ ${ }^{1}$ GuangDong Institute of Automation, Guangdong Provincial Key Laboratory of Modern Control Technology, Guangdong Modern control \\ technology with optical and electrical Public Laboratory, Guangzhou 510070, Guangdong, China \\ ${ }^{2}$ Shenzhen Huizhicheng Technology Co. Ltd, Shenzhen 518057, Guangdong, China
}

\begin{abstract}
Aiming at the double motor driving structure of precision air suspension platform, the control strategy of the system is studied under the condition of various mechanical vibration and electrical signal noise. Based on controllability and observability of systems, the optimal controller is designed to realize the fast rise and fast stability of the system. Simulation results verify the effectiveness and feasibility of the control strategy, and the system performance is optimal.
\end{abstract}

\section{Introduction}

At present, the guide mode of traditional rotary servo motor combined with ball screw driven in precision motion platform has been gradually replaced by non friction supporting technology and non friction direct drive technology. The flotation technology oriented and bearing support form precision air motion platform is gradually in the field of high speed high accuracy servo motion emerge [1], [2], which directly driven by linear motor for the way to eliminate the system friction and dead zone nonlinear disturbance and structure with no mechanical contact, can achieve sub micron or even nanometer positioning accuracy in the long travel range.

Precision air suspension platform has the advantages of high precision, no friction, low pollution and other advantages, which are widely used in the field of lithography technology, ultra precision machining, detection of biological technology, nano surface topography measurement, and to continue to develop in the direction of high speed, high acceleration, high accuracy [3], [4]. But due to the lack of damping mechanism, direct drive vulnerable to interference and rigid flexible coupling is weak link characteristics will undoubtedly increase the difficulty of controller design. Erkorkmaz et al. [5] use the input shaping and state feedback controller to suppress the residual vibration of the air bearing platform and realize the precision control of the air bearing platform. Kim et al. [6] Combine with the approximate time optimal control and the delay control, which apply to the precision air suspension platform to suppress the residual vibration of the platform and reduce the stability time of the Jung-Jae. [7] and [8] respectively in the application of fuzzy algorithm and neural network algorithm to suppress the residual vibration and vibration control system by input shaping technique.

Although these control algorithms have certain compensation to the system, but the system performance also affected. At the same time, air suspension motion platform for external disturbance, (such as linear motor force ripple, drive electrical noise and measurement noise, cable of cable force), change the environment and the change of system parameters will directly affect the platform movement precision. Therefore, this paper for precision air suspension platform of double motor drive structure, in the presence of external disturbances, the system of modeling and optimal control strategy are designed, so as to achieve the system's rapid rise and rapid and stable, the performance of the system to achieve optimal.

\section{System Modeling}

The single axis motion of the double motor drive in a precision air suspension motion platform is described by a dual input dual output linear system,

$$
\left\{\begin{array}{c}
x(k+1)=A_{c} x(k)+B_{c} u_{c}(k)+B_{c, w} w(k) \\
y_{c}(k)=C_{c} x(k)+v(k)
\end{array}\right.
$$

The system is affected by the internal and external environment of the system. Form in, $k \in Z^{+}$is the number of time steps, the system state variable is the displacement and speed of the two ends of the push rod: $x=\left[x_{1}, x_{2}, v_{1}, v_{2}\right]^{T}$. System control vector is input voltage signal: $u=\left[u_{1}, u_{2}\right]^{T}$, The system output vector 
is the displacement of the two ends of the push rod: $y=\left[y_{1}, y_{2}\right]^{T}$.

$$
w=\left[w_{1}, \cdots, w_{n}\right]^{T} \in R^{n}, v=\left[v_{1}, \cdots, v_{p}\right]^{T} \in R^{p}
$$

are external disturbance and random noise vector. Assume:

(1) $w(k)$ and $v(k)$ are the zero mean Gauss white noise vector for each other, and they are independent of each other:

$E\{w(k)\}=0 \quad, \quad \operatorname{Cov}\{w(k), w(\tau)\}=Q_{k} \delta(k-\tau) \quad ;$ $E\{v(k)\}=0 \quad, \quad \operatorname{Cov}\{v(k), v(\tau)\}=R_{k} \delta(k-\tau) \quad ;$ $\operatorname{Cov}\{w(k), v(\tau)\}=0$.

Form in, $E(\bullet)$ is mathematical expectation; $Q_{k}$ is non negative definite symmetric matrix for corresponding dimension, which is the covariance matrix of $w(k) ; R_{k}$ is the positive definite symmetric matrix of the corresponding dimension, which is the variance matrix of $v(k) ; \quad \delta(t-\tau)$ is the Kronecker $\delta$ function, that is,

$$
\delta(t-\tau)= \begin{cases}1, & t=\tau \\ 0, & t \neq \tau\end{cases}
$$

(2) The initial value $x(0)$ of the state vector $x(k)$ is a random variable. The statistical properties of $x(0)$ are known, that is,

$$
E\{x(0)\}=x_{o}, E\left\{\left[x(0)-x_{o} \llbracket\left[x(0)-x_{o}\right]^{T}\right\}=P(0)\right.
$$

(3) $x(0)$ and $w(k), v(k)$ are independent of each other, that is,

$$
\operatorname{Cov}\{w(k), x(0)\}=0, \operatorname{Cov}\{v(k), x(0)\}=0
$$

\section{Synchronous optimal control problem description}

According to the establishment of the dynamic model (1), the optimal control problem for the precision air suspension platform with disturbance and noise can be expressed as:

$$
u_{c}(k)=-L(k) \cdot x(k)=-L(k) \cdot \hat{x}(k)
$$

Because the system state variable $x(k)$ is the displacement and speed of the push rod, $x_{1}$ and $x_{2}$ can be directly measured by the precision grating ruler, and the speed term $v_{1}$ and $v_{2}$ can not be directly measured. Therefore, it is needed to design the algorithm to estimate the value of the algorithm. The optimal control problem of the system is changed into:

$$
u_{c}(k)=-L(k) \cdot\left[\begin{array}{c}
x_{1}(k) \\
x_{2}(k) \\
\hat{v_{1}}(k) \\
\hat{v_{2}}(k)
\end{array}\right]
$$

That is to solve the stochastic optimal control rate makes the random two performance indicators to achieve the minimum:

$$
J=E\left[x^{T}(N) Q_{1} x(N)+\sum_{k=0}^{N-1}\left(x^{T}(k) Q_{2} x(k)+u_{c}^{T}(k) R u_{c}(k)\right)\right]
$$

In the formula, $Q_{1}, Q_{2}$ are the state vector weighting matrix, which are the symmetric nonnegative definite matrix. $\mathrm{R}$ is the input vector weighted matrix, which is the symmetric positive definite matrix.

According to the formula of the optimal control rate (2), the control strategy can be divided into two parts: the optimal estimation of the state vector and the optimal gain of the system, as shown in Fig. 1.

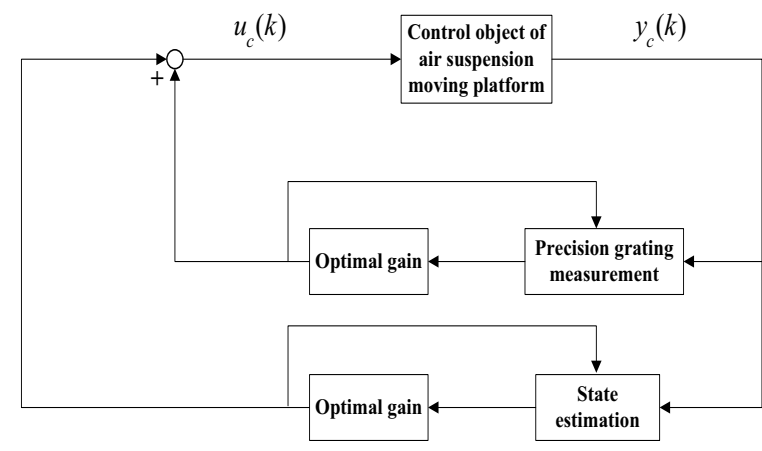

Figure 1. Synchronous optimal controller based on double motor drive precision air suspension platform

\section{Synchronous optimal controller design}

\subsection{System controllability and observability}

According to the dual motor driven precision air suspension platform system model, if the system matrix $A_{c}$ is non singular matrix, and at time $k \in\left[0, t_{f}\right]$ range: $\left.\operatorname{rank} \mid\left(A_{c}\left(t_{f}-1\right) A_{c}\left(t_{f}-2\right) \cdots A_{c}(1)\right) B_{c}(0),\left(A_{c}\left(t_{f}-1\right) \cdots A_{c}(2)\right) B_{c}(1) \cdots, B_{c}\left(t_{f}-1\right)\right]=n$ and

$$
\operatorname{rank}\left[\begin{array}{c}
C_{c}(0) \\
C_{c}(1) \cdot A_{c}(0) \\
\cdots \ldots \\
C_{c}\left(t_{f}-1\right) \cdot\left(A_{c}\left(t_{f}-1\right) \cdots A_{c}(0)\right)
\end{array}\right]=n \quad, \quad \text { the }
$$

control system is completely controllability and observability.

Therefore, the optimal control strategy of the system is designed under the premise that the control system can be controlled and can be observed.

\subsection{Optimal state estimation $\hat{x}(k)$}

According to the observation sequence $y_{c}(0), y_{c}(1), \cdots$, $y_{c}(k)$, the motion velocity terms in the state vector 
$x(k)$ need to be estimated in order to achieve synchronization optimal control, which should be made as close as possible to the actual value, to reduce the synchronization error. Subjected to external interference of double motor driving precision gas suspension system model (1), through the discrete time Kalman filtering method of optimal state estimation $\hat{x}(k)$ is,

$$
\hat{x}(k)=\hat{x}(k \mid k-1)+K(k)\left[y_{c}(k)-C_{c}(k) \cdot \hat{x}(k \mid k-1)\right]
$$

In the formula, $K(k)$ is the optimal filtering gain, $\hat{x}(k \mid k-1)$ is one step optimal linear prediction estimation of state vector $x(k)$, which is obtained based on the system state equation.

$$
\hat{x}(k \mid k-1)=A_{c}(k-1) \hat{x}(k-1)+B_{c}(k-1) u_{c}(k-1)
$$

As a result, the filter estimation error of the state vector $x(k)$ is:

$$
\begin{aligned}
\tilde{x}(k) & =x(k)-\hat{x}(k) \\
& =x(k)-\hat{x}(k \mid k-1)-K(k)\left[y_{c}(k)-C_{c}(k) \hat{x}(k \mid k-1)\right] \\
& =\tilde{x}(k \mid k-1)-K(k) C_{c}(k) \tilde{x}(k \mid k-1)-K(k) v(k)
\end{aligned}
$$

According to the orthogonality theorem, the estimation error $\tilde{x}(k)$ should be orthogonal to the measured value $y_{c}(k)$ :

$$
\begin{aligned}
& E\left\{\tilde{x}(k) y_{c}{ }^{T}(k)\right\}=E\left\{\tilde{x}(k \mid k-1)-K(k) C_{c}(k) \tilde{x}(k \mid k-1)\right. \\
& \left.-K(k) v(k)\left[C_{c}(k) x(k)+v(k)\right]^{T}\right\} \\
& =E\left\{\tilde{x}(k \mid k-1) x^{T}(k) C_{c}^{T}(k)-K(k) C_{c}(k)\right. \\
& \left.\cdot \tilde{x}(k \mid k-1) x^{T}(k) C_{c}^{T}(k)-K(k) R_{k}\right\}=0
\end{aligned}
$$

The optimal filter gain matrix:

$$
\left.K(k)=P(k \mid k-1) C_{c}^{T}(k) C_{c}(k) P(k \mid k-1) C_{c}^{T}(k)+R_{k}\right]^{-1}
$$

Form in,

$P(k \mid k-1)=E\left\{\tilde{x}(k \mid k-1) \tilde{x}^{T}(k \mid k-1)+\tilde{x}(k \mid k-1) \hat{x}^{T}(k \mid k-1)\right\}$

Which is the optimal estimation error variance matrix of state vector $x(k)$. Because the optimal estimation error is:

$$
\tilde{x}(k \mid k-1)=x(k)-\hat{x}(k \mid k-1)=A_{c}(k-1) \tilde{x}(k-1)+B_{c, w}(k-1) w(k-1)
$$

Then,

$$
\begin{aligned}
& \mathrm{P}(\mathrm{k} \mid \mathrm{k}-1)=\mathrm{E}\left\{\tilde{x}(k \mid k-1) \tilde{x}^{T}(k \mid k-1)\right\}=\mathrm{E}\left\{\left[\mathrm{A}_{\mathrm{c}}(\mathrm{k}-1) \tilde{x}(k \mid k-1)\right.\right. \\
& \left.\left.+B_{c, w}(k-1) w(k-1)\right]\left[\mathrm{A}_{\mathrm{c}}(\mathrm{k}-1) \tilde{x}(k \mid k-1)+B_{c, w}(k-1) w(k-1)\right]^{\mathrm{T}}\right\}
\end{aligned}
$$

That is,

$P(k \mid k-1)=A_{c}(k-1) P(k-1 \mid k-1) A_{c}{ }^{T}(k-1)+B_{c, w}(k-1) Q_{k} B_{c, w}{ }^{T}(k-1)$
Form in, $P(k-1 \mid k-1)$ is the estimate the error variance matrix for the optimal filter, that is,

$P(k-1 \mid k-1)=E\left\{\tilde{x}(k-1) \tilde{x} \tilde{x}^{T}(k-1)\right\}$

$=P(k-1 \mid k-2)-K(k-1) C_{c}(k-1) P(k-1 \mid k-2)-P(k-1 \mid k-2)$

$\cdot C_{c}^{T}(k-1) K^{T}(k-1)+K(k-1) C_{c}(k-1) P(k-1 \mid k-2) C_{c}^{T}(k-1) K^{T}(k-1)$

$+K(k-1) R_{k} K^{T}(k-1)$

Then,

$P(k \mid k)=P(k \mid k-1)-K(k) C c(k) P(k \mid k-1)-P(k \mid k-1) C_{c}^{T}(k) K^{T}(k)$

- $K(k) C_{c}(k) P(k \mid k-1) C_{c}^{T}(k) K^{T}(k)+K(k) R_{k} K^{T}(k)$

That is,

$P(k \mid k)=P(k \mid k-1)-P(k \mid k-1) C_{c}^{T}\left(k\left\lceil C_{c}(k) P(k \mid k-1) C_{c}^{T}(k)+R_{k}\right]^{-1} C_{c}^{T}(k) P(k \mid k-1)\right.$

According to the characteristics of the Kalman filter due to its in the calculation process does not need to store any data, but to continue to "predict revise" push calculated optimal state estimates recursively, and computation and observation of the Kalman gain matrix $K(k)$ is independent of the value of the. Therefore, the filter gain value can be calculated in advance to reduce the actual amount of calculation. Integrated (6), (7) and (5) to obtain the optimal estimation error variance matrix recursion relations are as follows:

$$
\begin{aligned}
& P(k+1 \mid k)=A_{c}(k) P(k \mid k-1) A_{c}^{T}(k)+B_{c, w}(k) Q_{k} B_{c, w}{ }^{T}(k)-A_{c}(k) P(k \mid k-1) \\
& \cdot C_{c}^{T}\left(k\left\lceil C_{c}(k) P(k \mid k-1) C_{c}{ }^{T}(k)+R_{k}\right]^{-1} C_{c}{ }^{T}(k) P(k \mid k-1) A_{c}{ }^{T}(k)\right.
\end{aligned}
$$

Formula (8) is called forward Riccati equation. Under the premise of ensuring the system can control and can view, step by step forward to obtain the steady solution of the forward Riccati equation according to the given initial value $P(0 \mid 0)$, and then the filter gain matrix $K(k)$ can be obtained off-line.

\subsection{Optimal gain $L(k)$}

After the optimal filter estimation of the state vector is obtained, the solution of the optimal gain matrix in the optimal control rate is considered. This problem can be solved by solving the linear quadratic optimal regulator for two times. In order to ensure the disturbed by the noise and the disturbance of the air suspension system can meet the performance optimal conditions, even if the random quadratic performance index reach the minimum, to achieve the rapid rise and rapid and stable, the optimal gain matrix $L(k)$ need satisfies the equation:

$$
L(k)=\left[B_{c}{ }^{T}(k) S(k+1) B_{c}(k)+R\right]^{-1} B_{c}{ }^{T}(k) S(k+1) A_{c}(k)
$$

In the formula, $S(\cdot)$ satisfies the dynamic backward Riccati equation (10): 


$$
\left\{\begin{array}{l}
S(N)=Q_{1} \\
S(k)=A_{c}{ }^{T}(k) S(k+1) A_{c}(k)+Q_{2} \\
-A_{c}{ }^{T}(k) S(k+1) B_{c}(k)\left[B_{c}{ }^{T}(k) S(k+1) B_{c}(k)+R\right]^{-1} B_{c}{ }^{T}(k) S(k+1) A_{c}(k)
\end{array}\right.
$$

In the formula, $Q_{1}$ and $Q_{2}$ are the state vector weighted matrix in the performance index, and $\mathrm{R}$ is the input vector weighted matrix in the performance index. $\mathrm{N}$ represents the termination time. Under the premise of ensuring the system can control and can observe, step by step forward iteration to get the stable solution of the backward Riccati equation based on the value of the given end time.

\section{Simulation analysis}

Based on the above mentioned above, a model of the air suspension system is established, considering a double input and double output discrete time system with a shape like (1), the initial state of each parameter and system is as follows:

$$
\begin{aligned}
A_{c} & =\left[\begin{array}{cccc}
0 & 0 & 1 & 0 \\
0 & 0 & 0 & 1 \\
0.1 & -0.3 & 0 & -0.25 \\
0 & -0.43 & 0.2 & -0.35
\end{array}\right], B_{c}=\left[\begin{array}{cc}
0 & 0 \\
0 & 0 \\
1 & 1 \\
201 & 218
\end{array}\right] \\
C_{c} & =\left[\begin{array}{llll}
1 & 0 & 0 & 0 \\
0 & 1 & 0 & 0
\end{array}\right], B_{c, w}=\left[\begin{array}{llll}
1 & 0 & 0 & 0 \\
0 & 1 & 0 & 0 \\
0 & 0 & 1 & 0 \\
0 & 0 & 0 & 1
\end{array}\right]
\end{aligned}
$$

System disturbance: $w(k) \sim N\left(0, I_{4 * 4}\right)$;

Random measurement noise: $v(k) \sim N\left(0, I_{2 * 2}\right)$;

Initial state vector: $x(0)=[100,100,0,0]^{T}$;

State estimate initial value:

$$
\hat{x}(0)=\left[\begin{array}{llll}
97.12 & 49.3 & 0.05 & 0.05
\end{array}\right]^{T} \text {; }
$$

Filtering estimation error variance initial value:

$$
p(0)=4 I_{4 * 4} \text {; }
$$

State vector weighted matrix: $Q_{1}=Q_{2}=25 I_{4 * 4}$; Input vector weighted matrix: $R=I_{2 * 2}$.

According to the system parameters and initial conditions given above, after 7 steps of iteration, the stability of the forward Riccati equation (8) is obtained:

$$
\mathrm{P}(8)=\left[\begin{array}{cccc}
2.1023 & 0.1382 & -0.0098 & 0.2076 \\
0.1382 & 2.2282 & -0.2661 & -0.3538 \\
-0.0098 & -0.2661 & 1.1243 & 0.1674 \\
0.2076 & -0.3538 & 0.1674 & 1.2830
\end{array}\right] \text { Based on }
$$

the solution of the optimal estimation error variance matrix $P(\cdot)$, the kalman filter gain matrix $K(k)$ is calculated:

$$
K=\left[\begin{array}{cc}
0.7603 & -0.2253 \\
-0.2397 & 0.7747 \\
0.0311 & -0.0934 \\
0.1230 & -0.1530
\end{array}\right]
$$

At the same time, after 2 steps of iteration, the stable solution of the backward Riccati equation (10) is:

$$
S(3)=\left[\begin{array}{cccc}
25.0098 & -0.0294 & -0.0000 & 0.0245 \\
-0.0294 & 25.0882 & 0.0000 & 0.0735 \\
-0.0000 & 0.0000 & 50.0098 & -0.0294 \\
-0.0245 & 0.0735 & -0.0294 & 50.1494
\end{array}\right]
$$

The periodic solution $S(\cdot)$ into (9), the solutions of optimal gain for linear quadratic regulator:

$$
L=\left[\begin{array}{ccrl}
1.2572 & -3.7463 & -0.0117 & -3.1225 \\
-1.1592 & 3.4522 & 0.0117 & 2.8774
\end{array}\right]
$$

Based on the filter gain and optimal gain of linear quadratic regulator, the optimal state estimation $\hat{x}(k)$ can be further calculated according to the formula (3) and (4), which is shown in Figure 1,and the output of the system corresponding and control sequences such as shown in Fig. 2.

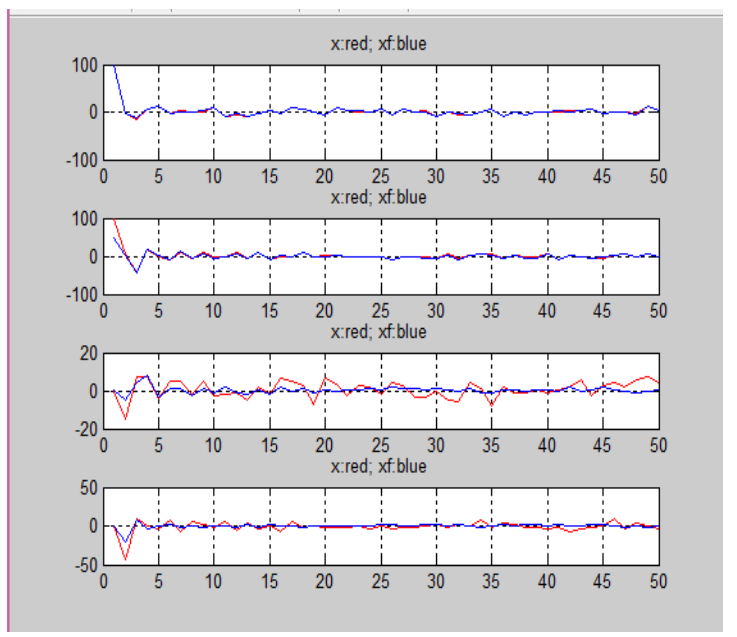

Figure 2. State vector and optimal state vector estimation

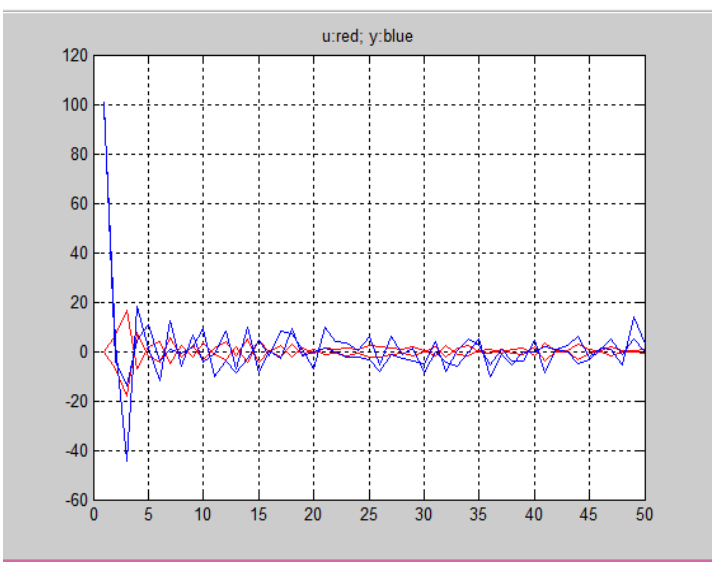

Figure 3. System output response and control sequence diagram.

It can be seen from the change trend of the curve in the chart, for a multi input and multi output air suspension system, the external disturbance has a certain effect on the control performance of the system. However, the state estimates eventually in the vicinity of the zero tends to be stable, which are estimated by the Kalman filtering algorithm. And the error between the estimated value and the actual state value is controlled within a very 
small range. The simulation results show that the compensation effect of Calman filtering algorithm guarantees the stability of the system to a certain extent, and it also ensures the precision accuracy of the control action.

\section{Conclusion}

In this paper, a dual motor drive structure for precision air suspension platform is established, and the mathematical model of the system is established under the condition of various mechanical vibration and electrical noise. Under the premise of ensuring the control system can be controlled and be observed, the design of the optimal controller is designed to realize the fast rising and fast stabilization of the system. The effectiveness and feasibility of the control strategy are verified by simulation experiment. Simulation experimental results show that the design of synchronous optimal control algorithm for a variety of system disturbance and random noise with certain compensation, makes the system has been able to maintain relatively stable output, and has high control performance.

\section{References}

1. WANG ying. high-acceleration/high-precision motion control of linear servo system for IC packing [D].Shanghai: Shanghai Jiao Tong University(2006)

2. PU donglin. Rapid vibration suppression and high precision positioning control of high acceleration air bearing motion system [D].Shanghai: Shanghai Jiao Tong University(2011)

3. LI yuntang. Study on high-acceleration/highprecision aerostatic positioning stage for IC packing [D]. Shanghai: Shanghai Jiao Tong University(2007)

4. DONG zeguang. Modeling, analysis and control of a gas-lubricated precision positioning stage [D]. Shanghai: Shanghai Jiao Tong University(2014)

5. B.K. Yoo, W.C. Ham, Adaptive control of robot manipulator using fuzzy compensator, IEEE Transactions on Fuzzy Systems, 8(2) , 186-199(2000)

6. H. A. Talebi, R.V. Patel and K. Khorasani, Control of flexible-link manipulators using neural networks, Springer Press(2001)

7. Erkorkmaz K, Gorniak J, Gordon D, Precision machine tool XY stage utilizing a planar air bearing arrangement[J]. CIRP Annals-Manufacturing Technology, 59(1):425-428(2010)

8. Viktorov V, Belforte G, Raparelli T, Modeling and identification of gas journal bearings: Externally pressurized gas bearing results[J]. Journal of tribology, 127(3):548-556(2005) 